\title{
Abstract \#122901
}

\section{Fabrication of Super Hydrophobic Gas Diffusion Layers}

D. S. Thumbarathy and P. K. Das (University of Newcastle upon Tyne)

\section{Abstract Text:}

Gas diffusion layer (GDL) plays a significant role in water management and heat removal within the polymer electrolyte fuel cells (PEFCs). When the GDL is flooded with liquid water, PEFC undergoes mass transport losses due to pore blockage by water which leads to decreased cell performance. For this purpose, the fabrication of selective wetting pattern by spray coating on the GDL surface is reported in this study. The present study will investigate the surface morphology of the GDL determined by its pore geometry and hydrophobicity which can control the water transport in PEFCs. Commercial GDLs were selected as a substrate. Two different monomers were used and their emulsions of required concentration were spray coated and polymerised to achieve the hydrophobic coatings. Regardless to monomer's viscosity, super hydrophobic coatings on Commercial GDLs were obtained via spray coating with a contact angle of $159^{\circ}$ or higher, while the base surface's contact angle was in the range of $100^{\circ}$.

The fabricated GDLs were further analysed by means of sliding angles and scanning electron microscopy. Fourier Transform Infrared Spectroscopy (FTIR) analysis confirmed the successful introduction of the functional groups in both the coatings. Pore size distribution and adhesion forces measurement were used to investigate the water droplet transport on the coated GDLs. In this presentation, the results of our study will be discussed with the aim of highlighting how these results will benefit the water management of next generation high-power PEFCs.

\section{Symposium Selection:}

Mass and Charge Transfer across Electrochemical Interfaces - Session 3

Slot:

: Thursday, 25 July 2019: 14:00-17:00

\section{Scheduled Time:}

$15: 40$

Submitter's E-mail Address:

deepashree.thumbarathy@newcastle.ac.uk

Preferred Presentation Format:

Oral

First Corresponding Author

Dr. Deepashree Sudhir Thumbarathy

Affiliation(s): University of Newcastle upon Tyne

Address:

School of Engineering (Mechanical)

Ground Floor, Stephenson Building

Newcastle University

Newcastle Upon Tyne, NE1 7RU

United Kingdom

Phone Number: +44 (0) 1912087340

E-mail Address: deepashree.thumbarathy@newcastle.ac.uk

Second Author

Dr. Prodip K Das

Affiliation(s): University of Newcastle upon Tyne

Phone Number: +4401912086170

E-mail Address: prodip.das@newcastle.ac.uk 